\title{
Frailty and Risk of Cardiovascular Diseases in Older Persons: The Age, Gene/Environment Susceptibility-Reykjavik Study
}

\author{
Nicola Veronese, Kristin Sigeirsdottir, Gudny Eiriksdottir, Elisa A. Marques, ${ }^{3}$ Didier Chalhoub, \\ Caroline L. Phillips, Lenore J. Launer, Stefania Maggi, Vilmundur Gudnason,, ${ }^{3}$ and Tamara B. Harris ${ }^{3}$
}

\begin{abstract}
Frailty is a risk factor for cardiovascular diseases (CVD), but the studies available have not considered the presence of subclinical atherosclerotic disease as potential confounders. We investigated the association between frailty and the onset of CVD independently of subclinical atherosclerotic disease. For this reason, a sample of 3818 older participants participating in the Age, Gene/Environment Susceptibility-Reykjavik Study without CVD at baseline was followed for a median of 8.7 years. Frailty was defined as the presence of $\geq 3$ among five Fried's criteria (unintentional weight loss, low physical activity level, weakness, exhaustion, and slow gait speed). Incident CVD was defined as onset of coronary artery disease, heart failure, stroke, and CVDrelated mortality identified using hospital, medical, and death records. Subclinical atherosclerotic disease was evaluated as the maximum value of carotid intima media thickness, presence of carotid plaque (moderate or high), and total coronary calcifications (CACs). At baseline, frail participants $(n=300)$ were more frequently obese, diabetic, and had a greater presence of metabolic syndrome than the nonfrail $(n=3518)$. Frail participants also showed a higher presence of carotid plaques and CACs. Using a Cox's regression analysis, adjusted for clinical, biochemical, and subclinical atherosclerosis estimates, frailty increased the risk of CVD (hazard ratio $[\mathrm{HR}]=1.35 ; 95 \%$ confidence interval $[\mathrm{CI}]: 1.05-1.74)$, with results stronger for women than men $(\mathrm{HR}=1.51$, $p=0.006$ and 1.19, $p=0.44$, respectively). Among Fried's criteria, exhaustion was the only criterion significantly associated with the onset of new CVD events ( $\mathrm{HR}=1.30 ; 95 \% \mathrm{CI}: 1.00-1.73)$. In conclusion, frailty was associated with the onset of CVD in older people even after adjusting for subclinical atherosclerotic disease.
\end{abstract}

Keywords: cardiovascular disease, risk factor, aging, frailty

\section{Introduction}

$\mathbf{F}$ RAILTY IS USUALly DEFINED as a state of reduced physiological reserve and increased vulnerability for poor resolution of homeostasis after a stressor event. ${ }^{1}$ This condition is highly prevalent in subjects with cardiovascular diseases (CVD), reaching more than $50 \%$ in some studies. ${ }^{2}$

In a large study involving more than 40,000 older women, coronary artery disease (CAD) and stroke were the most important predictors of frailty. ${ }^{3}$ Other studies substantially con- firmed these findings. ${ }^{4,5}$ Moreover, it is well known that the presence of frailty in older adults with CVD increases the risk of falls, institutionalization, repeated hospitalization, and mortality. ${ }^{6}$

Since frailty and CVD share common conditions such as chronic low-grade inflammation ${ }^{7}$ and insulin resistance, ${ }^{1}$ recent research proposed that frailty could be considered a potential CVD risk factor. ${ }^{8-11}$ However, most of these studies have not included an evaluation of subclinical atherosclerotic disease using imaging techniques. These subclinical measures

\footnotetext{
${ }^{1}$ National Research Council, Neuroscience Institute-Aging Branch, Padova, Italy.

${ }^{2}$ Icelandic Heart Association, Kopavogur, Iceland.

${ }^{3}$ Laboratory of Epidemiology, and Population Sciences, National Institute on Aging, Bethesda, Maryland.

${ }^{4}$ University of Iceland School of Medicine, Reykjavik, Iceland.
}

(c) Nicola Veronese et al., 2017; Published by Mary Ann Liebert, Inc. This is an Open Access article distributed under the terms of the Creative Commons Attribution License, which permits unrestricted use, distribution, and reproduction in any medium, provided the original work is properly cited. 
could affect the association between frailty and incident CVD since frail people seem to have a higher prevalence of silent carotid plaques and higher intima media thickness (IMT), ${ }^{9}$ as well as more abnormal ankle-brachial index, carotid stenosis, and left ventricular hypertrophy than those without frailty. ${ }^{12} \mathrm{On}$ the contrary, frailty could be associated with the onset of CVD independently from subclinical atherosclerotic disease through several mechanisms, including higher oxidative stress level ${ }^{13}$ and genetic alterations, such as reduction in telomere length. ${ }^{14}$

As a result, whether the higher incidence of CVD found in older frail people could be due to the presence of these subclinical atherosclerotic abnormalities or if frailty is independent of these early atherosclerotic alterations is not well understood.

Therefore, the aim of the present prospective study was to investigate the impact of frailty (defined using the most common definition proposed by Linda Fried ${ }^{15}$ ) on the risk of developing CVD in a cohort of community-dwelling older men and women adjusting for baseline subclinical atherosclerotic disease. We hypothesized that frail individuals would still have a significant risk of developing CVD after controlling the presence of subclinical atherosclerotic disease at the baseline, and their risk would be higher compared with nonfrail older adults.

\section{Materials and Methods}

\section{Study population}

The Age, Gene/Environment Susceptibility (AGES)Reykjavik Study is a population-based study of 5764 subjects older than 65 years from the 40-year-long Reykjavik Study. Full details of the investigations are described in the study's baseline article. ${ }^{16}$ Subjects underwent extensive laboratory and imaging investigations, including a detailed evaluation of subclinical atherosclerotic measures.

\section{Medical conditions and biochemical examinations assessment}

Depressive symptoms were assessed through the 15-item geriatric depression scale (GDS) with a score $\geq 6$ used to indicate the presence of clinically relevant depressive symptoms. ${ }^{17}$ The presence of chronic obstructive pulmonary disease was assessed through self-reported questionnaire and the use of medications, while the presence of atrial fibrillation (AF) was assessed through electrocardiography (ECG) and hospital records. Hypertension was defined by self-report of treatment with antihypertensive drugs, mean systolic blood pressure $\geq 140$, or mean diastolic blood pressure $\geq 90 \mathrm{mmHg}$. Diabetes was assessed by self-report with concomitant use of antidiabetic medications, HbA1c $\geq 6.5 \%$, or fasting plasma glucose (FPG) $\geq 126 \mathrm{mg} / \mathrm{dL}$. Metabolic syndrome was defined as the presence of at least three among five according to the National Cholesterol Education Program Adult Treatment Panel III criteria. Renal function was estimated through the eGFR using the Modification of Diet in Renal Disease formula. High-sensitivity C-reactive protein (CRP) was measured on a Hitachi 912. Both within- and between-assay quality control procedures were used, and the coefficient of variation of the method was $1.3 \%$ $3.4 \%$, respectively, through the period of data collection. The assay could detect a minimal CRP concentration of $0.1 \mathrm{mg} / \mathrm{L}$, and values below this level were classified as undetectable.

\section{Measures of subclinical atherosclerosis}

The subclinical atherosclerotic measures were based on common carotid IMT and carotid plaque severity measured on ultrasound images and calcium in the coronary arteries measured on computer tomography (CT) images.

Ultrasound images were acquired with a Sequoia C256 (Siemens Medical Systems, Erlangen, Germany). Standard B-mode images of the carotid IMT were acquired for the predefined segment of each common carotid artery (CCA; right and left) at defined interrogation angles using Meijers arc. Standard images were obtained from four angles at each site. The mean IMT of the near and far walls were determined from a single image at each interrogation angle for both the right and left CCA. The maximum value of all these IMT measures was used in this work. Complete details about how to measure IMT are available elsewhere. ${ }^{18}$

The presence of atherosclerotic lesions of the left and right carotid bifurcation and internal carotid artery (carotid plaques) was quantified on line during the ultrasound examination. The most severe lesions per segment were assessed in a semiquantitative manner and categorized as moderate/severe versus none/minimal lesions. ${ }^{19}$

Images for calcium scoring were acquired using a Siemens Somatom Sensation 4 Multidetector CT (Siemens Medical Solutions, Erlangen, Germany) with prospective ECG triggering. The entire heart and thoracic aorta were scanned sequentially in two separate acquisitions in the cranio-caudal direction during suspended inspiration. Calcium in the coronary arteries was quantified using the Agatston method. Coronary calcification (CAC) was expressed as a sum score for all four coronary arteries. Interobserver variability assessment showed high average correlation between the five readers and an expert Multi-Ethnic Study of Atherosclerosis (MESA) reader $(r=0.94)$ based on the reanalysis of 200 scans. A cutoff of 400 Agatston units was taken as suggestive of the presence of CAC. ${ }^{20}$ The CAC analysis technique is extensively described elsewhere. ${ }^{21}$

The data about IMT and carotid plaques were not available for 81 participants. Moreover, 178 and 46 participants did not have data for carotid plaques/IMT and CAC estimation, respectively.

\section{Exposure definition}

Fried defined frailty using five measurable items (unintentional weight loss, low physical activity level, weakness, exhaustion, and slow gait speed). ${ }^{15}$

- Unintentional weight loss $(n=75$ at baseline): selfreported weight loss of $5 \%$ or more in the past 12 months without known reasons.

- Self-reported low physical activity level $(n=559)$ : less than 4 hours per week of moderate or vigorous physical activities in the past 12 months.

- Weakness $(n=879)$ : average handgrip strength value of three attempts of the dominant hand below the sex and body mass index (BMI) specific cutoffs suggested by Fried et al. ${ }^{15}$

- Exhaustion ( $n=209)$ : a GDS score $\geq 6$ and a negative answer to the question "Do you feel full of energy?",17

- Slow gait speed $(n=549)$ : the best timed walk over $6 \mathrm{~m}$ at usual pace below the sex- and height-specific cutoffs. $^{15}$ 
Participants were classified as frail if they met at least three criteria. Subjects unable to perform the handgrip strength $(n=4)$ or walking tests $(n=198)$ for physical or mental problems were considered as having weakness or slow gait speed, respectively. ${ }^{15}$

\section{Outcomes definition}

Prevalent and incident CAD was defined as a history of revascularization, hospitalization for myocardial infarction, electrocardiographic evidence of myocardial infarction, or self-reported history of CAD accompanied by the use of antiangina medications. Stroke was defined as a documented (e.g., from medical documentation) history of stroke or through hospital records. Heart failure (HF) was defined on the basis of hospital records. Mortality from CVD (codes from 390 to 459 according to International Classification of Diseases (ICD)-9th Revision, 2002 and codes from I00 to I99 in ICD-10th Revision, 2010) was also included until December 2009. A copy of the official death certificate of all deceased participants was obtained.

\section{Statistical analyses}

For continuous variables, normal distributions were tested using the Shapiro-Wilk test. If normality was satisfied, data were presented using means ( \pm standard deviations); if nonnormal, the data were modeled as medians with interquartile range (IQR). Counts and percentages were used for categorical variables. Age- and gender-adjusted $p$-values were calculated using a general linear model for continuous variables and a logistic regression for categorical ones.

Cox's proportional hazard models were used to assess the association between frailty and the onset of CVD. Known factors associated with frailty and/or CVD were considered for inclusion in the analysis. To explore whether a variable should be included, the log-rank test of equality across strata was performed for the categorical variables and Cox's univariate proportional hazards regression for the continuous variables. The predictors included in the final models were all the variables reaching a $p<0.10$ in the univariate analyses or those variables that were significantly different among frail and nonfrail participants $(p<0.05)$.We built three models to assess the role of the covariates in the association between frailty and CVD onset: Model 1 was adjusted for age and sex; Model 2 was additionally adjusted for baseline clinical and biochemical confounders; and Model 3 was adjusted for the confounders in the Models 1-2 and for the presence of subclinical atherosclerotic disease parameters. Due to the large number of covariates, a logistic regression backward analysis was applied in all three models.

Collinearity among covariates was quantified using the variance inflation factor, taking a cutoff of two as exclusion criterion. BMI, high-density lipoproteins, low-density lipoproteins, triglycerides, and FPG were excluded having a high collinearity with waist circumference, serum cholesterol levels, and diabetes presence, respectively. Hazard ratios (HRs) and 95\% confidence intervals (CIs) were used to compare CVD incidence in frail vs. nonfrail participants.

To test the robustness of our findings, we conducted several sensitivity analyses stratifying for all clinical factors independently associated with CVD onset in the univariate analyses, but only gender resulted a significant moderator of this association. In these analyses, continuous variables were categorized according to the median value of the sample included in this survey.

HRs were also calculated for specific frailty criteria, using the same covariates as in the final model and taking participants without any Fried's criteria as reference. All analyses were performed using the SPSS 21.0 for Windows (SPSS, Inc., Chicago, Illinois). All statistical tests were twotailed, and statistical significance was assumed for a $p$-value $<0.05$

\section{Results}

Among the 5764 participants initially involved in the AGES study, $155(=2.6 \%)$ participants were excluded based on not having data about frailty, $56(=1.0 \%)$ because they lacked data on CVD, and $1735(=31.0 \%)$ because of a CVD diagnosis at baseline. The participants with CVD at baseline were older $(78.0 \pm 5.7$ vs. $76.2 \pm 5.6$ years, $p<0.0001)$, and a greater proportion of them were males $(58.0 \%$ vs. $36.0 \%$, $p<0.0001)$. CVD participants reported an almost doubled presence of frailty (15\%) than those without CVD (8\%).

\section{Baseline characteristics}

The analytical sample consisted of 3818 older participants (mean age $=76.2 \pm 5.6$ years; women $=64 \%$ ) with a mean BMI of $27.0 \pm 4.5 \mathrm{~kg} / \mathrm{m}^{2}$ and no CVD at the baseline.

Table 1 shows the baseline characteristics by presence of frailty. The 300 frail participants were older and more frequently women than those without frailty $(n=3518)$. After adjusting for age and sex, frail subjects were more depressed, obese, diabetic, and had higher prevalence of metabolic syndrome. Regarding biochemical examinations, frail subjects had higher serum CRP levels and worse renal function than those without frailty. Frail participants had more moderate or severe carotid plaque $(75.6 \%$ vs. $62.0 \%, p=0.01)$ and CACs $(43.8 \%$ vs. $32.6 \%, p=0.01)$ than those without frailty. However, there were no statistically significant differences in the use of low-dose aspirin or statins (Table 1).

\section{Follow-up data}

Over a median period of 8.7 years $(\mathrm{IQR}=5.2-9.8$ years $)$, 999 cardiovascular events (451 in males and 548 in women equal to $45.1 \%$ and $54.9 \%$ of the baseline population) were recorded (654 developed CAD, 296 stroke, $187 \mathrm{HF}$, and 203 participants died of CVD) with an age- and sexadjusted incidence rate of 41 events (95\% CI: 9-169) per 1000 person-years.

The cumulative incidence of CVD in frail participants was higher than in those without frailty $(38.9 \%$ vs. $25.0 \%$, $p<0.0001$ ) as reported in Figure 1.

As shown in Table 2, using a Cox's regression analysis fully adjusted for variables, including clinical, biochemical, and subclinical atherosclerosis parameters (Model 3), frailty increased the risk of developing a CVD event during follow-up $(\mathrm{HR}=1.35 ; 95 \% \mathrm{CI}: 1.05-1.74, p=0.02)$. The relationship between frailty and CVD events resulted in gender differences ( $p$ for interaction frailty by gender $=0.02$ ) being statistically significant in women $(\mathrm{HR}=1.51 ; 95 \% \mathrm{CI}: 1.12-2.03, p=$ $0.006)$ and not in men $(\mathrm{HR}=1.19 ; 95 \%$ CI: $0.77-1.85$, 
Table 1. Baseline Characteristics by Frailty Status

\begin{tabular}{|c|c|c|c|}
\hline Participants' characteristics & Frail $(\mathrm{n}=300)$ & Nonfrail $(\mathrm{n}=3518)$ & $\mathrm{p}^{\mathrm{a}}$ \\
\hline Age (years) & $79.5 \pm 6.1$ & $75.9 \pm 5.4$ & $<0.0001^{\mathrm{b}}$ \\
\hline Female sex $(n, \%)$ & $228(73.3)$ & $2204(62.8)$ & $<0.0001^{\mathrm{c}}$ \\
\hline \multicolumn{4}{|l|}{ General characteristics } \\
\hline Current smokers $(n, \%)$ & $37(12.0)$ & $432(12.6)$ & 0.36 \\
\hline Dependent in ADL $(n, \%)^{\mathrm{c}}$ & 119 (39.4) & $2717(19.3)$ & $<0.0001$ \\
\hline GDS (score) & $4.5 \pm 3.1$ & $2.0 \pm 1.7$ & $<0.0001$ \\
\hline MMSE (score) & $25.3 \pm 4.0$ & $26.8 \pm 3.0$ & $<0.0001$ \\
\hline $\mathrm{SBP}(\mathrm{mmHg})$ & $142.5 \pm 22.2$ & $142.5 \pm 20.5$ & 0.11 \\
\hline $\mathrm{DBP}(\mathrm{mmHg})$ & $73.3 \pm 11.0$ & $74.2 \pm 9.6$ & 0.39 \\
\hline \multicolumn{4}{|l|}{ Anthropometric characteristics } \\
\hline BMI $\left(\mathrm{kg} / \mathrm{m}^{2}\right)$ & $28.1 \pm 5.3$ & $26.9 \pm 4.4$ & $<0.0001$ \\
\hline Waist circumference $(\mathrm{cm})$ & $104.3 \pm 13.3$ & $100.0 \pm 12.0$ & $<0.0001$ \\
\hline \multicolumn{4}{|l|}{ Medical conditions and drugs } \\
\hline Atrial fibrillation $(n, \%)$ & $22(7.1)$ & $142(4.0)$ & 0.10 \\
\hline $\operatorname{COPD}(n, \%)$ & $33(10.6)$ & $335(9.6)$ & 0.57 \\
\hline Hypertension $(n, \%)$ & $228(74.0)$ & $2418(69.2)$ & 0.53 \\
\hline Diabetes $(n, \%)$ & $46(14.8)$ & $381(10.9)$ & 0.01 \\
\hline Metabolic syndrome $(n, \%)$ & $114(36.9)$ & $997(28.4)$ & $<0.0001$ \\
\hline Use of antihypertensives $(n, \%)$ & $179(57.6)$ & $1619(46.2)$ & $<0.0001$ \\
\hline Use of low-dose aspirin $(n, \%)$ & $81(26.0)$ & $783(22.3)$ & 0.34 \\
\hline Use of lipid-lowering drugs $(n, \%)$ & $33(10.6)$ & $390(11.1)$ & 0.91 \\
\hline \multicolumn{4}{|l|}{ Biohumoral tests } \\
\hline $\mathrm{CRP}(\mathrm{mg} / \mathrm{L})$ & $2.2(1.1-4.9)$ & $1.8(0.9-3.7)$ & $<0.0001$ \\
\hline FPG (mmol/L) & $5.73 \pm 1.30$ & $5.7 \pm 1.02$ & 0.35 \\
\hline $\mathrm{HbA} 1 \mathrm{c}(\%)$ & $5.70 \pm 0.58$ & $5.66 \pm 0.48$ & 0.33 \\
\hline $\mathrm{eGFR}(\mathrm{mL} / \mathrm{min})$ & $68.8 \pm 19.1$ & $69.4 \pm 16.8$ & 0.04 \\
\hline Total cholesterol (mmol/L) & $5.63 \pm 1.10$ & $5.80 \pm 1.11$ & $<0.0001$ \\
\hline $\mathrm{HDL}(\mathrm{mmol} / \mathrm{L})$ & $1.52 \pm 0.42$ & $1.64 \pm 0.43$ & $<0.0001$ \\
\hline $\mathrm{LDL}(\mathrm{mmol} / \mathrm{L})$ & $3.56 \pm 0.96$ & $3.70 \pm 0.97$ & 0.04 \\
\hline Triglycerides (mmol/L) & $1.24 \pm 0.62$ & $1.17 \pm 0.57$ & 0.03 \\
\hline \multicolumn{4}{|l|}{ Subclinical atherosclerotic disease estimates } \\
\hline Maximum IMT (mm) & $1.14 \pm 0.15$ & $1.13 \pm 0.16$ & 0.78 \\
\hline Carotid plaque (moderate or higher) ${ }^{\mathrm{d}}$ & $214(75.6)$ & $2014(62.0)$ & 0.01 \\
\hline Coronary calcium (Agatston) & $298(64-902)$ & $171(22-568)$ & 0.01 \\
\hline Coronary calcifications & $131(43.8)$ & $1104(32.6)$ & 0.008 \\
\hline
\end{tabular}

Numbers are mean values ( \pm standard deviations), median (with interquartile range), or number and percentages (\%), as appropriate.

${ }^{a}$ Unless otherwise specified, $p$-values are adjusted for age and gender using a general linear model or logistic regression, as appropriate.

${ }^{\mathrm{b}}$ Not adjusted for age or gender, respectively.

${ }^{c}$ Dependency in ADL was defined as one or more limitations in the ADL.

${ }^{\mathrm{d}}$ At the most compromised carotid artery.

${ }^{\mathrm{e}}$ Presence of coronary calcifications was defined as a coronary calcium score $>400$ Agatston.

ADL, activities of daily living; BMI, body mass index; COPD, chronic obstructive pulmonary disease; CRP, C-reactive protein; DBP, diastolic blood pressure; eGFR, estimated glomerular filtration rate; FPG, fasting plasma glucose; GDS, geriatric depression scale; HbA1c, glycosylated hemoglobin; HDL, high-density lipoproteins; IMT, intima-media thickness; LDL, low-density lipoproteins; MMSE, MiniMental State Examination; SBP, systolic blood pressure.

$p=0.44)$ (Table 2). Other interactions between the presence of frailty and clinical, biochemical, and subclinical atherosclerosis estimates tested did not affect our results.

After testing each of the Fried's criteria individually, only exhaustion was associated with a higher risk of CVD $(\mathrm{HR}=1.30 ; 95 \% \mathrm{CI}: 1.00-1.73 ; p=0.05)$. The statistically significant association between the other Fried's criteria and CVD was removed after adjusting for subclinical atherosclerotic estimates (Table 2).

\section{Discussion}

In our cohort of community-living elderly people, the presence of frailty at baseline was associated with an in- creased risk of future CVD by about $35 \%$, after considering subclinical atherosclerotic alterations.

\section{Baseline characteristics of frail participants}

Frail participants had an unfavorable metabolic profile at the baseline as shown by higher presence of depression, obesity, diabetes, and metabolic syndrome. All these findings are in agreement with the current literature showing that $\operatorname{mood}^{22}$ and metabolic alterations ${ }^{23}$ are strongly associated with frailty. The presence of poorer renal function and higher serum CRP levels further confirmed this unfavorable phenotype. Therefore, the higher presence of subclinical atherosclerosis in frail older adults compared with 


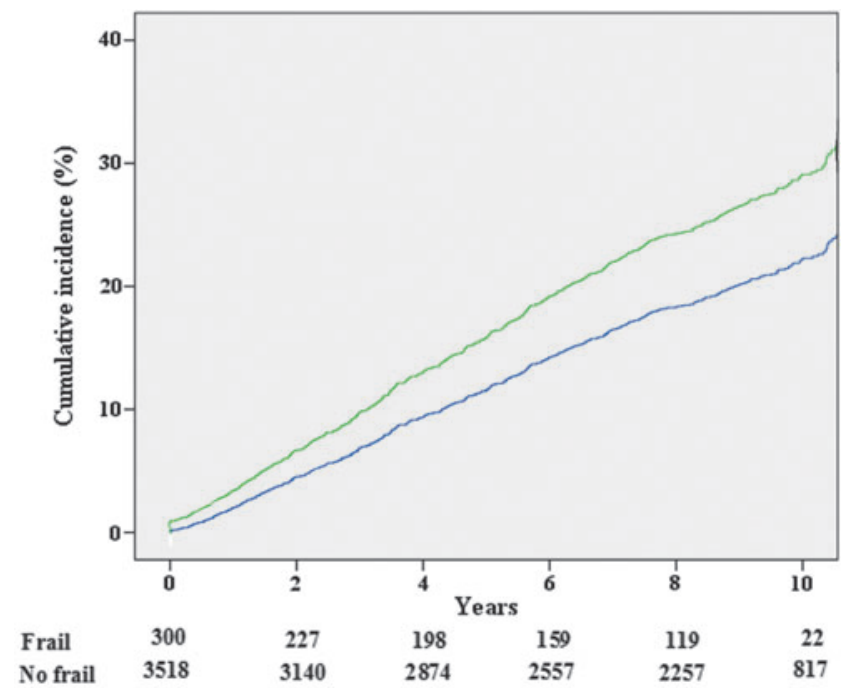

FIG. 1. Cumulative incidence of cardiovascular events by years of follow-up and presence of frailty at the baseline. Cumulative incidence curves based on adjusted Cox regression of cardiovascular events according to baseline presence of frailty. The numbers below the figure represent the number at risk of cardiovascular events according to presence or not of frailty at the baseline. Green line represents the cumulative incidence of frail participants, the blue one of no frail. Color images available online at www.liebertpub.com/rej

those without frailty was expected and was in agreement with other studies on this topic. ${ }^{12,24}$

\section{Frailty increases the risk of CVD}

After adjusting our analyses for traditional potential confounders (such as age, sex, and biochemical factors) and particularly measures of subclinical atherosclerotic disease, we found that the association between frailty and CVD remained significant. In accordance with our results, other prospective studies demonstrated a significant association between frailty and CVD onset. ${ }^{8,9,11}$ However, these studies did not include any estimation of subclinical atherosclerotic disease. This is important since it is possible that the heavier burden of CVD observed in frail subjects may have been related to a higher presence of subclinical atherosclerotic disease and not to frailty per se. Since CACs and carotid plaques are among the strongest predictors of CVD, our study further confirms that frailty is an independent CVD risk factor in older adults. The reason through which frailty increased the risk of CVD is not known, and the observational nature of our study allows us only to speculate regarding this point. However, it is possible that frailty could increase the precipitation of CVD events during follow-up period and/or accelerate the rate of disease accumulation from the baseline subclinical atherosclerotic disease. Unfortunately, since subclinical atherosclerotic disease was not evaluated during follow-up, we could not know which mechanism was the most important.

\section{Molecular and cellular pathways of the relationship between frailty and CVD}

The cellular and physiological impairments present in frail people $^{25}$ may contribute to explain the association between frailty and CVD. First, at the cellular level, frail people seem to have higher oxidative stress levels, ${ }^{26}$ marked deoxyribonucleic acid damage, ${ }^{27}$ and shorter telomere length. ${ }^{25}$ Current research has shown that these factors play an important role in predicting the onset of CVD. Second, frail people have elevated inflammatory markers, which are known (particularly CRP) to play a role in the development of CVD. ${ }^{28}$ Moreover, high inflammation levels correspond to higher markers of thrombosis that could play an additional role in CVD onset, although in this analysis, we did assess the importance of high-sensitivity CRP as a confounder. ${ }^{6}$ Finally, endocrine dysregulations present in frailty could play a role in the development of CVD. It was reported in large crosssectional studies that frail people have lower IGF- $1^{29}$ and sex hormone levels ${ }^{30}$ compared with healthy controls, which may increase CVD risk in the elderly. ${ }^{31,32}$

Table 2. Association Between Frailty Status and Risk of Cardiovascular Diseases at Follow-Up

\begin{tabular}{|c|c|c|c|c|c|c|}
\hline & $\begin{array}{l}\text { Model } 1 \text { hazard } \\
\text { ratio }(95 \% \text { CI })\end{array}$ & $\mathrm{p}$ & $\begin{array}{l}\text { Model } 2 \text { hazard } \\
\text { ratio }(95 \% \text { CI })\end{array}$ & $\mathrm{p}$ & $\begin{array}{c}\text { Model } 3 \text { hazard } \\
\text { ratio }(95 \% \text { CI })\end{array}$ & $\mathrm{p}$ \\
\hline Frailty $^{\mathrm{a}}$ & $1.60(1.27-2.02)$ & $<0.0001$ & $1.38(1.07-1.78)$ & 0.01 & $1.35(1.05-1.74)$ & 0.02 \\
\hline Men & $1.54(1.03-2.31)$ & 0.04 & $1.20(0.78-1.87)$ & 0.41 & $1.19(0.77-1.85)$ & 0.44 \\
\hline Women & 1.65 (1.24-2.19) & 0.002 & $1.47(1.07-2.02)$ & 0.002 & $1.51(1.12-2.03)$ & 0.006 \\
\hline Slow gait speed & $1.32(1.09-1.61)$ & 0.005 & $1.19(0.97-1.45)$ & 0.09 & $1.11(0.91-1.37)$ & 0.29 \\
\hline Unintentional weight loss & $1.45(0.92-2.29)$ & 0.11 & - & - & - & - \\
\hline Exhaustion & $1.48(1.12-1.95)$ & 0.006 & $1.37(1.05-1.82)$ & 0.03 & $1.30(1.00-1.73)$ & 0.05 \\
\hline Weakness & $1.24(1.05-1.46)$ & 0.01 & $1.17(0.99-1.38)$ & 0.07 & $1.15(0.98-1.36)$ & 0.10 \\
\hline Low physical activity & $1.09(0.89-1.33)$ & 0.43 & - & - & - & - \\
\hline
\end{tabular}

Unless otherwise specified, data are presented as hazard ratios and 95\% CIs. A logistic regression backward analysis was applied in all the models. Model 1 was adjusted for age (as continuous) and gender. Model 2 was adjusted for the covariates in Model 1 and baseline values of MMSE score (as continuous); waist circumference (as continuous); average SBP (as continuous); independency in ADL (yes/no); presence of atrial fibrillation, metabolic syndrome, use of antihypertensive medications, low-dose aspirin, and statins (all yes/no); serum levels of CRP (categorized in quartiles); eGFR, HbAlc, total cholesterol (all as continuous). Model 3 was adjusted for the covariates in the previous models and baseline maximum value of IMT (as continuous), carotid plaque (moderate or higher), presence of coronary calcifications (> 400 Agatston).

${ }^{a}$ The interaction frailty by gender taking incident CVD as outcome was significant for all the three models $(p<0.05)$.

$\mathrm{CI}$, confidence interval; CVD, cardiovascular diseases. 


\section{Gender differences}

Another remarkable point of our analyses is that frailty was associated with CVD onset in women, but not in men. The different effects of potential CVD risk factors on cardiovascular outcomes between sexes was recently observed in a large meta-analysis showing that diabetic women had a greater risk of stroke than men. ${ }^{33}$ These discrepancies may be explained by potential differences in diagnosis and treatment of CVD. In some countries, men at higher risk for CVD are diagnosed and pharmacologically treated earlier compared with women. ${ }^{34}$ Therefore, it is possible that a smaller number of frail women were treated compared with frail men, increasing the risk of CVD during follow-up. This hypothesis is indirectly confirmed by our results, showing that those with CVD at baseline were more common in men. However, lacking any specific data for Iceland, these issues remain largely theoretical.

\section{Single domains of frailty and CVD}

Since literature suggests that some characteristics strictly related to frailty (e.g., slow gait speed) are able to predict the onset of CVD per se, we have also investigated whether there exists a single Fried's criterion associated with the onset of CVD. This could be important also for a clinical point of view to reduce time in evaluating frailty in older people.

Among the single Fried's criterion, only exhaustion emerged as a significant predictor of CVD at follow-up. The presence of exhaustion carried a $30 \%$ higher risk of having a new CVD. This finding is in agreement with recent literature, showing an important role of depression and depressive mood in metabolic and CVD. ${ }^{35}$ Depressed people share several common pathways with CVD, including inflammation, hypercortisolemia, and increased adrenergic tone. ${ }^{36}$ Moreover, it is possible that some conditions leading to exhaustion (such as fatigue and sleep problems ${ }^{37}$ ) that are recently recognized as possible CVD risk factors could have contributed to this finding.

On the contrary, we failed to find any association between the other Fried's criteria taken singularly and the onset of CVD, particularly regarding slow gait speed that seems to be a statistically significant predictor in other studies. 9,36 At first, explanation could be that atherosclerosis may impair the physical activity tests, although only subclinically, ${ }^{38}$ more so than the mood. Therefore, the correction for atherosclerosis estimates reduced the association between poor scores on physical performance tests and the onset of new CVD. Second, it is possible that the prognostic importance of physical performance tests may depend on the demographic characteristics of the studied populations, and it would be more appropriate to use cutoffs tailored for each study to use these tests as prognostic markers. ${ }^{36}$ Altogether, these findings suggest that to take a single Fried's criterion as surrogate of frailty would not be appropriate, since important differences in definition and demographic characteristics could significantly influence the association between frailty and CVD.

\section{Limitations}

The findings of our study must be considered within its limitations. We did not assess the transitions of frail people during the follow-up period, but it could be of importance since the transition from frailty to disability could explain a part of our results. It could also be of interest to assess whether reversal of frailty could have a protective role for the onset of CVD. Moreover, weight loss was assessed by self-reported measures, and slowness and low physical activity levels were assessed using slightly different measures than the original criteria proposed by Fried. ${ }^{15}$ Adapting the frailty criteria can influence the quality of the composite score and potentially introduce bias. Third, the diagnosis of CVD could have some bias since a rigorous adjudication process was not performed. Fourth, at baseline, some participants did not make cardiovascular tests, and so, their data are missing. Even if, in the worst scenario, this percentage is very limited $(<5 \%)$, how much this lacking could affect our data is not detectable. Finally, some estimates of subclinical atherosclerotic disease often present in old people (such as alterations in ankle-brachial index) were not measured.

\section{Conclusion}

In conclusion, frailty was associated with the onset of CVD in older adults, even considering subclinical atherosclerotic measures. This association seems to be stronger in women than in men and in subjects affected by exhaustion. Our study further confirmed the importance of screening for frailty from a cardiological point of view to identify older adults who may be at increased risk for the onset of CVD, which in turn is related to increased risk of disability and mortality. More studies are needed to understand the impact of frailty treatment strategies in CVD risk in older adults and to explore the possible differences between men and women.

\section{Acknowledgments}

We thank the study participants and the Icelandic Heart Association clinic staff for their invaluable contribution.

\section{Financial Support}

This work was supported by National Institutes of Health (N01-AG-12100), the National Institute on Aging Intramural Research Program, Hjartavernd (the Icelandic Heart Association), and the Althingi (the Icelandic Parliament). E.A.M., D.C., L.J.L., and T.B.H. were supported, in part, by the Intramural Research Program of the National Institutes of Health, National Institute on Aging.

\section{Author Disclosure Statement}

No competing financial interests exist.

\section{References}

1. Clegg A, Young J, Iliffe S, Rikkert MO, Rockwood K. Frailty in elderly people. Lancet 2013;381:752-762.

2. Moriarty O, McGuire BE, Finn DP. The effect of pain on cognitive function: A review of clinical and preclinical research. Prog Neurobiol 2011;93:385-404.

3. Fugate Woods N, LaCroix AZ, Gray SL, Aragaki A, Cochrane BB, Brunner RL, Masaki K, Murray A, Newman AB. Frailty: Emergence and consequences in women aged 
65 and older in the women's health initiative observational study, J Am Geriatr Soc 2005;53:1321-1330.

4. Afilalo J, Alexander KP, Mack MJ, Maurer MS, Green P, Allen LA, Popma JJ, Ferrucci L, Forman DE. Frailty assessment in the cardiovascular care of older adults. J Am Coll Cardiol 2014;63:747-762.

5. Frisoli A, Ingham SJM, Paes AT, Tinoco E, Greco A, Zanata N, Pintarelli V, Elber I, Borges J, Camargo Carvalho AC. Frailty predictors and outcomes among older patients with cardiovascular disease: Data from Fragicor. Arch Gerontol Geriatr 2015;61:1-7.

6. Afilalo J. Frailty in patients with cardiovascular disease: Why, when, and how to measure. Curr Cardiovasc Risk Rep 2011;5:467-472.

7. Soysal P, Stubbs B, Lucato P, Luchini C, Solmi M, Peluso R, Sergi G, Isik AT, Manzato E, Maggi S, Maggio M, Prina AM, Cosco TD, Wu Y-T, Veronese N. Inflammation and frailty in the elderly: A systematic review and metaanalysis. Ageing Res Rev 2016;31:1-8.

8. Matsuzawa Y, Konishi M, Akiyama E, Suzuki H, Nakayama N, Kiyokuni M, Sumita S, Ebina T, Kosuge M, Hibi K, Tsukahara K, Iwahashi N, Endo M, Maejima N, Saka K, Hashiba K, Okada K, Taguri M, Morita S, Sugiyama $S$, Ogawa $H$, Sashika $H$, Umemura S, Kimura K. Association between gait speed as a measure of frailty and risk of cardiovascular events after myocardial infarction. J Am Coll Cardiol 2013;61:1964-1972.

9. Dumurgier J, Elbaz A, Ducimetière P, Tavernier B, Alpérovitch A, Tzourio C. Slow walking speed and cardiovascular death in well functioning older adults: Prospective cohort study. BMJ 2009;339:b4460.

10. Veronese N, Trevisan C, De Rui M, Bolzetta F, Maggi S, Zambon S, Corti MC, Baggio G, Perissinotto E, Crepaldi G, Manzato E, Sergi G. Serum dehydroepiandrosterone sulfate and risk for type 2 diabetes in older men and women: The Pro.V.A Study. Can J Diabetes 2016;40:158-163.

11. Veronese N, Cereda E, Stubbs B, Solmi M, Luchini C, Manzato E, Sergi G, Manu P, Harris T, Fontana L, Strandberg T, Amieva H, Dumurgier J, Elbaz A, Tzourio C, Eicholzer M, Rohrmann S, Moretti C, F. D’Ascenzo, Quadri G, Polidoro A, Lourenco RA, Moreira VG, Sanchis J, Scotti V, Maggi S, Correll CU. Risk of cardiovascular disease morbidity and mortality in frail and pre-frail older adults: Results from a meta-analysis and exploratory metaregression analysis. Ageing Res Rev 2017;35:63-73.

12. Newman AB, Gottdiener JS, Mcburnie MA, Hirsch CH, Kop WJ, Tracy R, Walston JD, Fried LP. Associations of subclinical cardiovascular disease with frailty. J Gerontol A Biol Sci Med Sci 2001;56:M158-M166.

13. Soysal P, Isik AT, Carvalho AF, Fernandes BS, Solmi M, Schofield P, Veronese N, Stubbs B. Oxidative stress and frailty: A systematic review and synthesis of the best evidence. Maturitas 2017;99:66-72.

14. Collerton J, Martin-Ruiz C, Davies K, Hilkens CM, Isaacs J, Kolenda C, Parker C, Dunn M, Catt M, Jagger C, von Zglinicki T, Kirkwood TBL. Frailty and the role of inflammation, immunosenescence and cellular ageing in the very old: Cross-sectional findings from the Newcastle 85+ Study. Mech Ageing Dev 2012;133:456-466.

15. Fried LP, Tangen CM, Walston J, Newman AB, Hirsch C, Gottdiener J, Seeman T, Tracy R, Kop WJ, Burke G, McBurnie MA. Frailty in older adults: Evidence for a phenotype. J Gerontol A Biol Sci Med Sci 2001;56:M146M156.
16. Harris TB, Launer LJ, Eiriksdottir G, Kjartansson O, Jonsson PV, Sigurdsson G, Thorgeirsson G, Aspelund T, Garcia ME, Cotch MF, Hoffman HJ, Gudnason V. Age, Gene/Environment Susceptibility-Reykjavik Study: Multidisciplinary applied phenomics. Am J Epidemiol 2007;165: 1076-1087.

17. Yesavage JA, Brink TL, Rose TL, Lum O, Huang V, Adey M, Leirer VO. Development and validation of a geriatric depression screening scale: A preliminary report. J Psychiatr Res 17:37-49.

18. Bots ML, Hofman A, Grobbee DE. Common carotid intima-media thickness and lower extremity arterial atherosclerosis. The Rotterdam Study. Arterioscler Thromb 1994; 14:1885-1891.

19. van der Meer IM, Bots ML, Hofman A, del Sol AI, van der Kuip DAM, Witteman JCM. Predictive value of noninvasive measures of atherosclerosis for incident myocardial infarction: The Rotterdam Study. Circulation 2004;109: 1089-1094.

20. Budoff MJ, Nasir K, McClelland RL, Detrano R, Wong N, Blumenthal RS, Kondos G, Kronmal RA. Coronary calcium predicts events better with absolute calcium scores than age-sex-race/ethnicity percentiles: MESA (MultiEthnic Study of Atherosclerosis). J Am Coll Cardiol 2009; 53:345-352.

21. Carr JJ, Nelson JC, Wong ND, McNitt-Gray M, Arad Y, Jacobs DR, Sidney S, Bild DE, Williams OD, Detrano RC. Calcified coronary artery plaque measurement with cardiac CT in population-based studies: Standardized protocol of Multi-Ethnic Study of Atherosclerosis (MESA) and Coronary Artery Risk Development in Young Adults (CARDIA) study. Radiology 2005;234:35-43.

22. Buigues C, Padilla-Sánchez C, Garrido JF, NavarroMartínez R, Ruiz-Ros V, Cauli O. The relationship between depression and frailty syndrome: A systematic review. Aging Ment Health 2015;19:762-772.

23. Sinclair A, Morley JE, Rodriguez-Mañas L, Paolisso G, Bayer T, Zeyfang A, Bourdel-Marchasson I, Vischer U, Woo J, Chapman I, Dunning T, Meneilly G, RodriguezSaldana J, Gutierrez Robledo LM, Cukierman-Yaffe T, Gadsby R, Schernthaner G, Lorig K. Diabetes mellitus in older people: Position statement on behalf of the International Association of Gerontology and Geriatrics (IAGG), the European Diabetes Working Party for Older People (EDWPOP), and the International Task Force of Experts in Diabetes. J Am Med Dir Assoc 2012;13:497-502.

24. Gharacholou SM, Tashiro T, Cha SS, Scott CG, Takahashi PY, Pellikka PA. Echocardiographic indices associated with frailty in adults $\geq 65$ years. Am J Cardiol 2015; Epub ahead of print. DOI: 10.1016/j.amjcard.2015.08.023.

25. Zaslavsky O, Cochrane BB, Thompson HJ, Woods NF, Herting JR, LaCroix A. Frailty: A review of the first decade of research. Biol Res Nurs 2013;15:422-432.

26. Mulero J, Zafrilla P, Martinez-Cacha A. Oxidative stress, frailty and cognitive decline. J Nutr Health Aging 2011;15: 756-760.

27. Ashar FN, Moes A, Moore AZ, Grove ML, Chaves PHM, Coresh J, Newman AB, Matteini AM, Bandeen-Roche K, Boerwinkle E, Walston JD, Arking DE. Association of mitochondrial DNA levels with frailty and all-cause mortality. J Mol Med (Berl) 2015;93:177-186.

28. Buckley DI, Fu R, Freeman M, Rogers K, Helfand M. Creactive protein as a risk factor for coronary heart disease: A systematic review and meta-analyses for the U.S. Pre- 
ventive Services Task Force. Ann Intern Med 2009;151: 483-495.

29. Cappola AR, Xue Q-L, Fried LP. Multiple hormonal deficiencies in anabolic hormones are found in frail older women: The Women's Health and Aging studies. J Gerontol A Biol Sci Med Sci 2009;64:243-248.

30. Hyde Z, Flicker L, Almeida OP, Hankey GJ, McCaul KA, Chubb SAP, Yeap BB. Low free testosterone predicts frailty in older men: The health in men study. J Clin Endocrinol Metab 2010;95:3165-3172.

31. Ren J, Anversa P. The insulin-like growth factor I system: Physiological and pathophysiological implication in cardiovascular diseases associated with metabolic syndrome. Biochem Pharmacol 2015;93:409-417.

32. Corona G, Rastrelli G, Monami M, Guay A, Buvat J, Sforza A, Forti G, Mannucci E, Maggi M. Hypogonadism as a risk factor for cardiovascular mortality in men: A meta-analytic study. Eur J Endocrinol 2011;165:687-701.

33. Peters SAE, Huxley RR, Woodward M. Diabetes as a risk factor for stroke in women compared with men: A systematic review and meta-analysis of 64 cohorts, including 775,385 individuals and 12,539 strokes. Lancet 2014;383: 1973-1980.

34. Krämer HU, Raum E, Rüter G, Schöttker B, Rothenbacher D, Rosemann T, Szecsenyi J, Brenner H. Gender disparities in diabetes and coronary heart disease medication among patients with type 2 diabetes: Results from the DIANA study. Cardiovasc Diabetol 2012;11:88.

35. Vancampfort D, Mitchell AJ, De Hert M, Sienaert P, Probst M, Buys R, Stubbs B. Type 2 diabetes in patients with major depressive disorder: A meta-analysis of prevalence estimates and predictors. Depress Anxiety 2015;32:763773.

36. Sergi G, Veronese N, Fontana L, De Rui M, Bolzetta F, Zambon S, Corti M-C, Baggio G, Toffanello ED, Crepaldi G, Perissinotto E, Manzato E. Pre-frailty and risk of cardiovascular disease in elderly men and women: The PRO.V.A. study. J Am Coll Cardiol 2015;65:976-983.

37. Wolk R, Gami AS, Garcia-Touchard A, Somers VK. Sleep and cardiovascular disease. Curr Probl Cardiol 2005;30: 625-662.

38. Hamer M, Kivimaki M, Lahiri A, Yerramasu A, Deanfield JE, Marmot MG, Steptoe A. Walking speed and subclinical atherosclerosis in healthy older adults: The Whitehall II study. Heart 2010;96:380-384.

Address correspondence to:

Nicola Veronese Neuroscience Institute-Aging Branch

DIMED

National Research Council Via Giustiniani Padova 2-35128

Italy

E-mail: ilmannato@gmail.com

Received: November 25, 2016

Accepted: October 10, 2017 